\title{
Lowest oscillating mode in a nanoscale planar waveguide with double-negative material
}

\author{
Min Cheng,' Yingwu Zhou,, Shangyuan Feng, Juqiang Lin,' and Rong \\ Chen $^{\text {b }}$ \\ ${ }^{a}$ Minjiang University, Department of Physics and Electronic Information Engineering, \\ Fuzhou, Fujian 350108, China \\ cm94@163.com \\ ${ }^{\mathrm{b}}$ Fujian Normal University, Key Laboratory of Optoelectronic Science and Technology for \\ Medicine, Ministry of Education, Fuzhou, Fujian 350007, China \\ chenr@fjnu.edu.cn
}

\begin{abstract}
The lowest oscillating waveguide mode can exist in guiding layer of a doublenegative material that is symmetrically encapsulated in a double-positive material, even when the guiding layer's thickness is many times smaller than the operating free-space wavelength.
\end{abstract}

Keywords: oscillating modes, nanoscale waveguides, double-negative material.

\section{INTRODUCTION}

An artificial material with both negative real permittivity and permeability, which is called the double-negative material (DNM), was experimentally demonstrated in 2001. Then, researchers have improved and scaled down these structures to fabricate a DNM at optical wavelengths [1], and have proposed metamaterials for various applications, such as electromagnetic cloaking, improved lenses, subdiffraction-limited light beams. For conventional waveguides based on total internal reflection, symmetrical waveguides do not have cutoff for $\mathrm{TE}_{0}$ or $\mathrm{TM}_{0}$ oscillating modes, and the dimension of the guiding layer can theoretically go close to zero. The fundamental oscillating mode does not exist in the DNM waveguide and it was shown that DNM waveguides support two classes of waves: oscillating and surface modes [2]. Recently, the subwavelength miniaturization of photonic components has been addressed by the use of metamaterials [3]. However, in Ref. 4 it is stated that as the thickness of guiding layer is reduced much below the working wavelength, all oscillating modes are eliminated and only surface modes are supported. Here we show that the first-order oscillating modes can exist even in the DNM waveguides with the thickness of the guiding layer 10 times smaller than the working wavelength; i.e., the guiding layers can be in nanometer scale.

\section{PRINCIPLE AND NUMERICAL RESULTS}

We consider a symmetric slab waveguide in a conventional planar geometry (see Fig. 1). In a general case, a slab of the thickness $d$ is made up of a DNM with relative permittivity $\varepsilon_{f}$ and relative permeability $\mu_{f}$, whose real parts are negative. We assume that the surrounding medium is DPM (double positive material), and therefore it is characterized by $\varepsilon_{c}$ and $\mu_{c}$, whose real parts are positive.

(C)2009 Society of Photo-Optical Instrumentation Engineers [DOI: 10.1117/1.3286425]

Received 2 Dec 2009; accepted 14 Dec 2009; published 15 Dec 2009 [CCC: 19342608/2009/\$25.00] Journal of Nanophotonics, Vol. 3, 039504 (2009) 


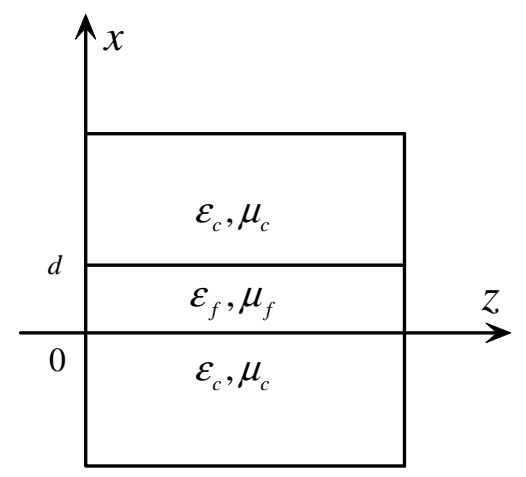

Fig. 1. Geometry of the problem.

We have derived analytical guided mode solutions of Maxwell's equations for this geometry. We searched for TE polarized monochromatic wave with an electric field of the form $\vec{E}_{y}=\vec{e}_{y} E_{0} \exp [i(\beta z-\omega t)]$, where $\omega$ is the angular frequency and $\beta$ is the propagation constant or the parallel component of the wave vector. A similar analysis can be carried out for the TM modes. Actually, by making the following corresponding transformations: $\left(\varepsilon_{f}, \mu_{f}, \varepsilon_{c}, \mu_{c}\right) \rightarrow\left(\mu_{f}, \varepsilon_{f}, \mu_{c}, \varepsilon_{c}\right)$ and $E_{y} \rightarrow H_{y}$, we can immediately obtain characteristic equations describing TM modes.

The mode profile $E_{0}$ in the three regions can be determined from the wave equation:

$$
E_{0}= \begin{cases}A \exp \left(-\kappa_{c} x\right), & \text { for } d<x<+\infty \\ B \exp \left(i k_{f} x\right)+C \exp \left(-i k_{f} x\right), & \text { for } 0<x<d, \\ D \exp \left(\kappa_{c} x\right), & \text { for }-\infty<x<0\end{cases}
$$

where $\kappa_{c}=\left(\beta^{2}-k_{0}^{2} \varepsilon_{c} \mu_{c}\right)^{1 / 2}, \gamma_{f}=\left(k_{0}^{2} \varepsilon_{f} \mu_{f}-\beta^{2}\right)^{1 / 2}, k_{0}=\omega / c=2 \pi / \lambda_{0}$, and $\lambda_{0}$ is the free-space wavelength. It is noted that $\kappa_{c}, \gamma_{f}, \beta$ should be positive. Matching the electric and magnetic fields at the boundaries yields the following dimensionless characteristic equation for oscillating modes in slab waveguides:

$$
\gamma_{f} d=2 \arctan \left(\kappa_{c} \mu_{f} / \gamma_{f} \mu_{c}\right)+m \pi
$$

where $m$ is the mode number. Since the sign of $\mu_{f} / \mu_{c}$ is negative, $m$ must be any integer greater than 0 . From the definition of $\kappa_{c}$ and $\gamma_{f}$, we have $k_{0}\left(\varepsilon_{c} \mu_{c}\right)^{1 / 2}<\beta<k_{0}\left(\varepsilon_{f} \mu_{f}\right)^{1 / 2}$. As $\beta$ approaches its minimum value (i.e., $k_{0}\left(\varepsilon_{c} \mu_{c}\right)^{1 / 2}$ ), Eq. (2) becomes

$$
d_{\min \beta}=\frac{m \lambda}{2 \sqrt{\varepsilon_{f} \mu_{f}-\varepsilon_{c} \mu_{c}}} .
$$

As $\beta$ approaches its maximum value (i.e., $k_{0}\left(\varepsilon_{f} \mu_{f}\right)^{1 / 2}$ ), $d$ approaches a finite value for $\mathrm{TE}_{1}$ mode,

$$
d_{\max \beta}=\left[(\pi / \lambda)\left|\mu_{f} / \mu_{c}\right| \sqrt{\varepsilon_{f} \mu_{f}-\varepsilon_{c} \mu_{c}}\right]^{-1},
$$


while it is infinite for all other TE modes. If $d_{\max \beta}$ of $\mathrm{TE}_{1}$ mode is less than $d_{\min \beta}$ of $\mathrm{TE}_{2}$ mode, the waveguide supports only the $\mathrm{TE}_{1}$ mode and the slab thickness $d$ is in an appropriate range:

$$
d=\frac{\pi+2 \arctan \left[\left(\mu_{f} / \mu_{c}\right)\left(\varepsilon_{f} \mu_{f} W^{2}-\varepsilon_{c} \mu_{c}\right)^{1 / 2} /\left(\varepsilon_{f} \mu_{f}-\varepsilon_{f} \mu_{f} W^{2}\right)^{1 / 2}\right]}{(2 \pi / \lambda)\left[\left(\varepsilon_{f} \mu_{f}\right)\left(1-W^{2}\right)\right]^{1 / 2}},
$$

where $W=\beta /\left[(2 \pi / \lambda)\left(\varepsilon_{f} \mu_{f}\right)^{1 / 2}\right]$ and $\left(\varepsilon_{c} \mu_{c} / \varepsilon_{f} \mu_{f}\right)^{1 / 2}<W<1$. From Eqs. (3) and (4) it is found that $d_{\min \beta}>d>d_{\max \beta}$ when $\pi / 2>\mu_{c} / \mu_{f}$ and $d_{\min \beta}<d<d_{\max \beta}$ when $\pi / 2<\mu_{c} / \mu_{f}$ for $\mathrm{TE}_{1}$ mode. According to Eqs. (3) and (4), it is clearly shown that $d_{\min \beta}$ and $d_{\max \beta}$ decrease with increasing of $R=\mu_{f} / \mu_{c}$.

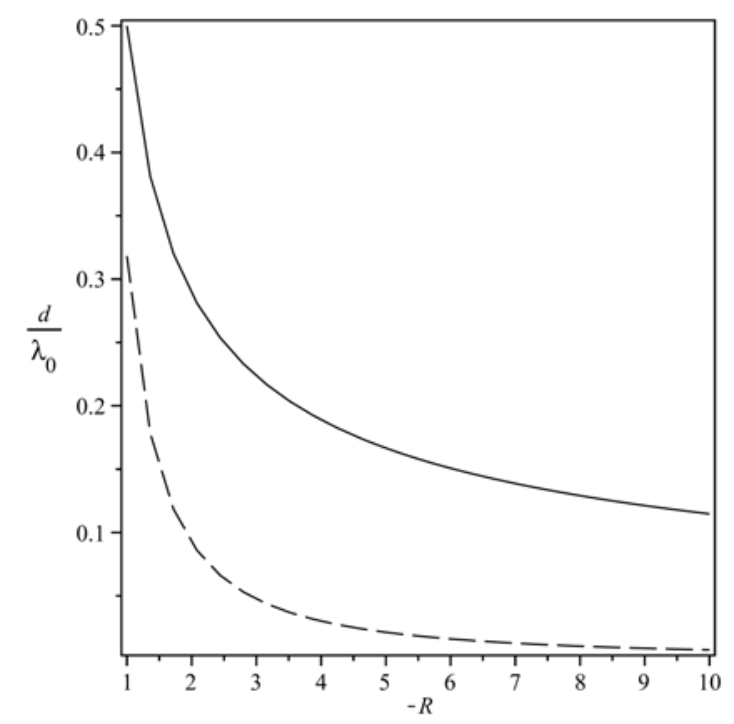

Fig. 2. $d_{\min \beta}$ (solid line) and $d_{\max \beta}$ (dashed line) as functions of $R$ for $\varepsilon_{c}=1, \mu_{c}=1$, and $\varepsilon_{f}=-2$.

Both $d_{\min \beta}$ (solid line) and $d_{\max \beta}$ (dashed line) are shown in Fig. 2 as functions of $R$ for $\varepsilon_{c}=1, \mu_{c}=1$, and $\varepsilon_{f}=-2$. Fig. 2 shows that $d_{\min \beta}$ and $d_{\max \beta}$ decrease with increasing of $R$. For $R>1.9013, d / \lambda_{0}$ could be less than 0.1 , implying that the light wave is in a guiding layer thinner than a tenth of the wavelength (i.e., waveguides with a nanoscale guiding layer for the $\mathrm{TE}_{1}$ oscillating mode can be developed). For $\varepsilon_{c}=1, \mu_{c}=1$, and $\varepsilon_{f}=-2$, Fig. 3 depicts the geometric dispersion curves for $\mathrm{TE}_{1}$ mode at different magnetic permeability ratios: $R=-5$ (solid line), -6 (dotted line), -9 (dashed line), -12 (dash-dotted line). For a certain value of the effective mode index $n_{\text {eff }}=\beta \lambda_{0} / 2 \pi$, we can see that the core thickness is small for high $-R$. It can be considered that the guiding layers can theoretically be scaled down as much as possible by increasing $-R$. 


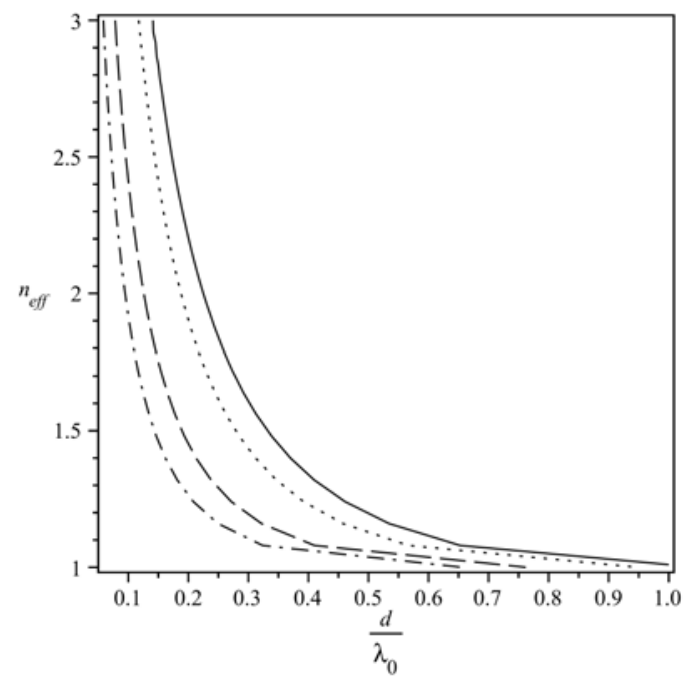

Fig. 3. The geometric dispersion curves for $\mathrm{TE}_{1}$ mode at different magnetic permeability ratios: $R=-5$ (solid line), -6 (dotted line), -9 (dashed line), -12 (dashdotted line). Other parameters are $\varepsilon_{c}=1, \mu_{c}=1, \varepsilon_{f}=-2$.

\section{DISCUSSION}

Remarkably, similar oscillating modes are supported by the inverted nanoscale structure, i.e., one with a DPM core and DNM claddings. Based on the explicit form of Eq.(2), it is inferred that all modal properties of the DPM-DNM-DPM arrangement, previously analyzed, are replicated by its "dual" counterpart. This property also provides increased flexibility in nanoscale waveguiding design utilizing DNM.

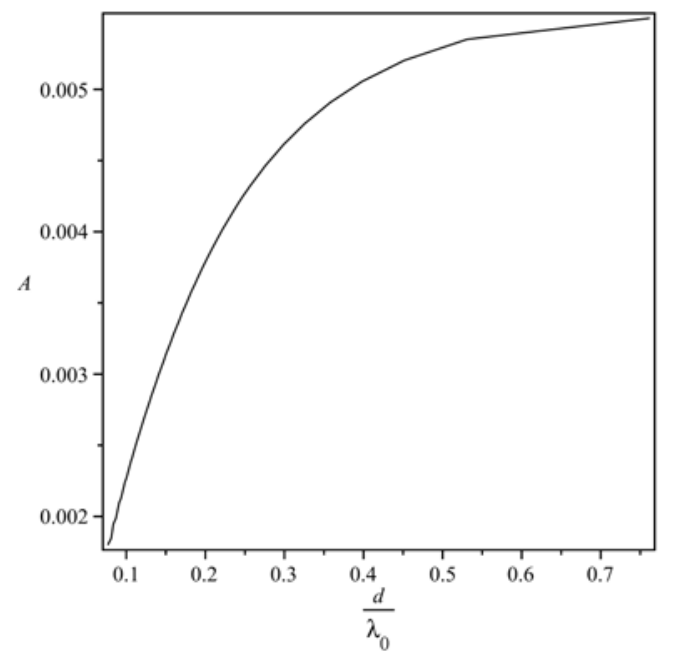

Fig. 4. $A=\operatorname{Im}\left(n_{\text {eff }}\right)=\alpha \lambda_{0} / 2 \pi$ is shown as a function of $d / \lambda_{0}$ for $\mathrm{TE}_{1}$ oscillating mode with $\varepsilon_{c}=1, \mu_{c}=1, \varepsilon_{f}=-2+0.001 i$, and $\mu_{f}=-9+0.001 i$. 
The role of losses is definitely very important in the studies of DNM. If DNM is a lossy medium, the effective mode index can be expressed as $n_{\text {eff }}=(\beta+i \alpha) \lambda_{0} / 2 \pi$, where $\alpha$ denotes the damping term. Here we assume that the losses are small. Using an analysis similar to that in Ref. 5, we get Fig. 4 where $A=\operatorname{Im}\left(n_{\text {eff }}\right)=\alpha \lambda_{0} / 2 \pi$ is shown as a function of $d / \lambda_{0}$ for the $\mathrm{TE}_{1}$ oscillating mode with $\varepsilon_{c}=1, \mu_{c}=1, \varepsilon_{f}=-2+0.001 i$, and $\mu_{f}=-9+0.001 i$. From Fig. 4 we can see that $\operatorname{Im}\left(n_{\text {eff }}\right)$ is slight when the guiding layer is thinner than the wavelength, i.e., the physical picture of the oscillating mode does not have significant changes except that the wave is propagating with attenuation along the waveguide.

\section{CONCLUSION}

In conclusion, we found that nanoscale planar waveguides with DNM can guide the firstorder oscillating modes. Although the surface modes allow operation on the nanometer scale, their inherent sensitivity to small variations of the media interfaces [6] may limit their practical use. For this reason, oscillating modes, which generally have their maxima inside the waveguide core, are more suitable for many applications. Applications for the waveguides proposed here include miniaturization of photonic integrated circuits, advancing their integration with nanophotonic, plasmonic, and electronic device elements.

\section{References}

[1] G. Dolling, C. Enkrich, M. Wegener, C. M. Soukoulis, and S. Linden, "Low-loss negative-index metamaterial at telecommunication wavelengths," Opt. Lett. 31, 1800-1802 (2006) [doi:10.1364/OL.31.001800].

[2] I. V. Shadrivov, A. A. Sukhorukov, and Y. S. Kivshar, "Guided modes in negativerefractive-index waveguides,” Phys. Rev. E 67, 057602 (2003) [doi: 10.1103/PhysRevE.67.057602].

[3] P. Tassin, X. Sahyoun, and I. Veretennicoff, "Miniaturization of photonic waveguides by the use of left-handed materials,” Appl. Phys. Lett. 92, 203111 (2009) [doi: 10.1063/1.2936299]

[4] Y. Satuby, N. Kaminsky, and M. Orenstein, "Nano-optical waveguide modes in gaps embedded in left-handed metamaterial," J. Opt. Soc. Am. B 24, A62-A68 (2007) [doi:10.1364/JOSAB.24.000A62].

[5] R. E. Collin, Field Theory of Guided Waves, 2nd ed, IEEE Press, Piscataway, NJ, USA (1991).

[6] K. L. Tsakmakidis, C. Hermann, A. Klaedtke, C. Jamois, and O. Hess, "Surface plasmon polaritons in generalized slab heterostructures with negative permittivity and permeability,” Phys. Rev. B 73, 085104 (2006) [ doi: 10.1103/PhysRevB.73.085104]. 